\title{
Static and dynamic characterization of droplets on hydrophobic surfaces
}

\author{
YAO ZhaoHui*, HAO PengFei, ZHANG XiWen \& HE Feng \\ School of Aerospace, Tsinghua University, Beijing 100084, China
}

Received October 9, 2011; accepted December 25, 2011

\begin{abstract}
We report on our study of the static and dynamic wetting property of hydrophobic surfaces with micro- and dual micro/nano-scale structures. Simulations based on the lattice Boltzmann method showed that the apparent contact angle of water droplets on hydrophobic surfaces with micro-scale structures increases as solid area decreases, whereas dual micro/nano-scale structures not only increase surface hydrophobicity but also greatly stabilize the Cassie state of droplets. Droplets falling on a superhydrophobic surface distort and, depending of free energy, sometimes bounced on the surface before finally adhering to the surface. These phenomena are in agreement with experimental observations. Simulated results also show that micro/nano-scale surface structures can increase droplet rebound height, which depends on static apparent contact angle.
\end{abstract}

droplet, apparent contact angle, hydrophobic surface, static/dynamic characterization, LBGK method

Citation: $\quad$ Yao Z H, Hao P F, Zhang X W, et al. Static and dynamic characterization of droplets on hydrophobic surfaces. Chin Sci Bull, 2012, 57: 1095-1101, doi: $10.1007 / \mathrm{s} 11434-012-5003-8$

As a result of natural selection, many adaptive features of organisms have approached varying degrees of perfection. More than 200 plant surfaces, such as that of the lotus leaf, have acquired the ability to self-clean, a trait that exploits surface superhydrophobicity [1]. Wetting phenomena depend on the free energy and geometry of surface structures $[2,3]$. In recent years, driven by potential applications including self-cleaning textiles, fluid transport in micro/nanoscale fluidics and bio-micro-electro-mechanical systems, various techniques have been developed to mimic lotus surfaces [4-6]. The static characteristics of water drops on hydrophobic surfaces have been studied experimentally [7-10]. Feng et al. [6] fabricated an array of carbon nanotubes that resembled real structures of a lotus leaf. From measurements, its apparent contact angle (ACA) was found to be $166^{\circ}$ with a sliding angle of $3^{\circ}$. In experiments of $\mathrm{Xu}$ and colleagues [11-13], droplets impacting on a solid surface were observed often to compress and rupture into tiny droplets on the flat surface. Reyssat et al. [14] found that

*Corresponding author (email: yaozh@tsinghua.edu.cn) merging of small satellite droplets can take place on textured superhydrophobic surfaces. However, they also found that water droplets impacting super-hydrophobic solids will often rebound. They discuss conditions in surface design (viz. those creating superhydrophobicity) under which such behavior can be observed. Using a high-speed CCD camera, Chen et al. [15] studied droplet-impact dynamics on various micro/nano-scaled surface structures and also performed static tests (ACA and sliding angle) on seven such surfaces. They found that a droplet hitting the nano-flower and the lotus leaf surfaces bounced. Minute satellite droplets were observed after impact from nano-flower surfaces, whereas no satellite droplets were observed for the lotus leaf. Bartolo and co-workers [16] reported three distinct regimes, in which the qualitative behavior (sticking, non-bouncing and bouncing) of an impacting droplet is different depending on the impact velocity.

In other studies [17,18], the level set and volume-of-fluid methods have been extended to model the moving contact lines. In [19], the droplet motions over grooved walls have been studied by the lattice Boltzmann method (LBM); there, 
the droplet was found to move faster on grooved hydrophobic surfaces than on hydrophilic counterparts. Hyvaluoma and Timonen [20] used a two-phase LBM to simulate droplet impacts on hydrophilic surfaces patterned with regular arrays of posts. Their results were in agreement with those of Bortolo et al. [16], i.e. the presence of three dynamic regimes depending on impact velocity. However, numerical simulations of droplet dynamics on dual micro/nano-scale surface structures have received less attention in the literature. The current study addresses this point.

\section{Simulation method}

The LBM, based on cellular-automata ideas (e.g. lattice gas), has emerged as a powerful tool for modeling complex fluid dynamics problems. In the simplest version of LBM, the Bhatnagar-Gross-Krook (BGK) approximation is used [21]. We refer the interested reader to Succi [22], who provided an excellent discussion and details on applying this BGK scheme.

Because three-dimensional computation needs a greater calculation mesh, a two-dimensional calculation is usually performed in simulations. A D2Q9 LBGK scheme proposed by Qian et al. [23] is used. The merit of this scheme is that it can easily deal with interactions between fluid and solid. The scheme is premised on setting nine 2-D particle velocities $\boldsymbol{e}_{i}$ each with corresponding particle-velocity distribution functions $f_{i}$. Accordingly, the macroscopic fluid density is

$$
\rho=\sum_{i=0}^{8} f_{i}
$$

The macroscopic velocity $\boldsymbol{u}$ is an average of the microscopic particle velocities $\boldsymbol{e}_{i}$ weighted by the velocity distribution functions $f_{i}$ :

$$
\boldsymbol{u}=\frac{1}{\rho} \sum_{i=0}^{8} \boldsymbol{e}_{i} f_{i}
$$

The evolution of the velocity distribution functions $f_{i}$ is

$$
f_{i}\left(\boldsymbol{r}+\boldsymbol{e}_{i} \Delta t, t+\Delta t\right)=f_{i}(\boldsymbol{r}, t)-\frac{\left[f_{i}(\boldsymbol{r}, t)-f_{i}^{\mathrm{eq}}(\boldsymbol{r}, t)\right]}{\tau},
$$

where $f_{i}(\boldsymbol{r}, t)$ is the velocity distribution function at the initial or last time step, $f_{i}\left(\boldsymbol{r}+\boldsymbol{e}_{i} \Delta t, t+\Delta t\right)$ the velocity distribution function at the new time step, and $\tau$ the collision time. The equilibrium distribution function $f_{i}^{\text {eq }}$ used in the D2Q9 algorithm is defined as

$$
f_{i}^{\mathrm{eq}}(r, t)=w_{i} \rho(r)\left[1+3 \frac{\boldsymbol{e}_{i} \cdot \boldsymbol{u}_{\mathrm{eq}}}{c^{2}}+\frac{9}{2} \frac{\left(\boldsymbol{e}_{i} \cdot \boldsymbol{u}_{\mathrm{eq}}\right)^{2}}{c^{4}}-\frac{3}{2} \frac{\boldsymbol{u}_{\mathrm{eq}}^{2}}{c^{2}}\right],
$$

where the weights $w_{i}$ are 4/9 for rest particles $(i=0), 1 / 9$ for $i=1-4$, and $1 / 36$ for $i=5-8 ; c$ is the basic speed on the lattice. The equilibrium velocity $\boldsymbol{u}_{\mathrm{eq}}$ can be written as

$$
\boldsymbol{u}_{\mathrm{eq}}=\boldsymbol{u}+\Delta \boldsymbol{u}=\boldsymbol{u}+\frac{\tau \boldsymbol{F}}{\rho}=\boldsymbol{u}+\frac{\tau\left(\boldsymbol{F}_{\mathrm{f}}+\boldsymbol{F}_{\mathrm{s}}\right)}{\rho}
$$

where $\boldsymbol{F}$ includes two kinds of interaction force $\boldsymbol{F}_{\mathrm{f}}$ and $\boldsymbol{F}_{\mathrm{s}}$. $\boldsymbol{F}_{\mathrm{f}}$ is an attractive force between neighboring fluid particles, and can be expressed as

$$
\boldsymbol{F}_{\mathrm{f}}(\boldsymbol{x}, t)=-G_{\mathrm{f}} \psi(\boldsymbol{x}, t) \sum_{\alpha=1}^{8} w_{\alpha} \psi\left(\boldsymbol{x}+\boldsymbol{e}_{\alpha} \Delta t, t\right) \boldsymbol{e}_{\alpha},
$$

where $G_{\mathrm{f}}$ is the interaction strength, $w_{\alpha}$ is $1 / 9$ for $\alpha=1-4$, $1 / 36$ for $\alpha=5-8$, and $\psi$ is the interaction potential:

$$
\psi=1-\exp (-\rho)
$$

$\boldsymbol{F}_{\mathrm{s}}$ is an adhesive interaction between fluid particles and solid surfaces. The strength of the force contribution, specified by an adsorption coefficient $G_{\mathrm{s}}$, is described in a similar form to $\boldsymbol{F}_{\mathrm{f}}$ :

$$
\boldsymbol{F}_{\mathrm{s}}(\boldsymbol{x}, t)=-G_{\mathrm{s}} \psi(\boldsymbol{x}, t) \sum_{\alpha=1}^{8} w_{\alpha} s w i\left(\boldsymbol{x}+\boldsymbol{e}_{\alpha} \Delta t\right) \boldsymbol{e}_{\alpha},
$$

where swi is a "switch" that takes value one if the site at $\boldsymbol{x}+\boldsymbol{e}_{\alpha} \Delta t$ is a solid and zero otherwise. The weights $w_{\alpha}$ are the same direction-dependent weighting factors as for $\boldsymbol{F}_{\mathrm{f}}$.

\section{Code test}

Using the above LBGK scheme, computer code has been developed to run simulations of droplet-on-surface impacts and analyze static and dynamic characteristics. In each simulation, a droplet with a diameter of 140 lattice spacings was positioned above a surface; $G_{\mathrm{f}}$ is set to -5.8 , and $G_{\mathrm{s}}$ to -1.1 . With these conditions, the droplet ACA on the flat surface is $135^{\circ} \pm 1.5^{\circ}$, as depicted in Figure 1(a). To test the correctness of the program, we simulated a droplet on a surface composed of micro-structures. The ACA in the heterogeneous regime can be expressed by the Cassie and Baxter equation [2]:

$$
\cos \theta_{\mathrm{C}}=f_{\mathrm{s}} \cos \theta_{\mathrm{Y}}-f_{\mathrm{v}},
$$

where $\theta_{\mathrm{C}}$ and $\theta_{\mathrm{Y}}$ are the respective droplet ACAs on rough and flat surfaces made of the same material; $f_{\mathrm{s}}$ and $f_{\mathrm{v}}$ are the respective fractions of solid and vapor under the droplet. By the Cassie and Baxter formula, the ACA on the micro-structured surface with solid-air fraction of 0.5556 should be $146.8^{\circ}$. From our simulation, resulting in Figure 1(b), the ACA of the droplet on this surface is $143^{\circ} \pm 1.5^{\circ}$, verifying that the code reproduces this important static characteristic. 

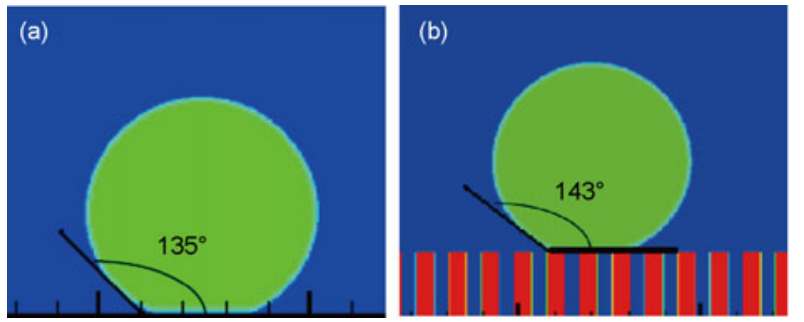

Figure 1 Static apparent contact angle for a droplet on flat and micro-structured surfaces $\left(G_{\mathrm{s}}=-1.1\right)$. (a) Flat surface; (b) surface with micro-ridges $\left(f_{\mathrm{s}}=0.556\right)$.

\section{Results and discussion}

With the tested code, we simulated the static droplet ACA on surfaces with various roughnesses. Additionally, we studied droplet impact dynamics on flat surfaces and two different micro-scale structured surfaces, and dual micro/nano-scale structured surfaces. The system size in simulations was $500 \times 500$ lattice spacings; the micro-ridge dimension was $10 \times 40$, and the distance between adjacent ridges was 4 or 8 lattice spacings. A droplet with a diameter of 140 lattice spacings was positioned on the surface.

\subsection{Static apparent contact angle}

In simulations, droplets of the same size were gently released from the same height. Figure 2 shows the variation of the static ACA for different structured surfaces with the same $G_{\mathrm{s}}=G_{\mathrm{s} 1}=-2.9$. For a smooth surface, the ACA is $95^{\circ} \pm 1.5^{\circ}$, thus the hydrophobicity is small. The ACAs of surfaces with micro-ridges increased to $105^{\circ} \pm 1.5^{\circ}$ and $120^{\circ} \pm 1.5^{\circ}$ (solid area fraction $f_{\mathrm{s}}$ is 0.714 and 0.556 , respectively). The ACA of the surface with dual-scale micro/nano structures increases to $130^{\circ} \pm 1.5^{\circ}$. These results agreed with a theoretical ACA formula [6] based on the triadic Koch curve from fractal geometry. From Figure 2, some observations can be made. For a hydrophobic surface, the presence of micro-textures seems to enhance the natural hydrophobicity of the surface. For a hydrophobic surface with microstructures, the smaller the solid area fraction (i.e. increasing micro-ridge spacings), the larger the ACA and the more hydrophobic the surface is. However, from Figure 1(b) and (c), it can be seen that the Cassie state of a droplet on a surface with smaller solid area fraction would gradually change to a Wenzel state. Along the bottom edge of the droplet, more liquid fills the micro-spaces nearer the edge midpoint that at the extremities. This is possibly caused by higher pressures on the bottom interface between thaw water and air. From Figure 1(d), we can see that the surface with dual micro/nano-scale structures, would not only increase the hydrophobicity of the surface, but also make the Cassie state of a droplet more stable. Our simulation results validated the conclusion that the hierarchical roughness makes superhydrophobic states stable, as proposed by other
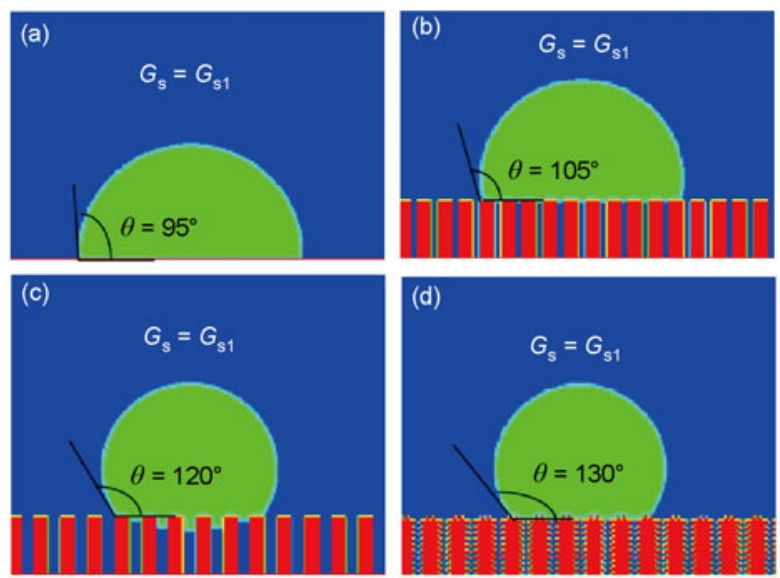

Figure 2 Static apparent contact angle for different structured surfaces $\left(G_{\mathrm{s}}=G_{\mathrm{s} 1}=-2.9\right)$. (a) Flat surface; (b) surface with micro-ridges $\left(f_{\mathrm{s}}=0.714\right)$; (c) surface with micro-ridges $\left(f_{\mathrm{s}}=0.556\right)$; (d) surface with dual-scale micro/nano structures.

researchers [15] in their experimental studies and theoretical analyses. These results can be used to explain why natural superhydrophobic surfaces have rough two-scale hierarchical structures: one micro-scaled, the other nano-scaled.

\subsection{Droplet impact dynamics}

As a droplet falls onto a surface with micro-ridges $\left(f_{\mathrm{s}}=\right.$ $\left.0.556, G_{\mathrm{s}}=G_{\mathrm{s} 1}=-2.9\right)$, it becomes distorted and finally adheres to the surface (see Figure 3). In this case, the surface is not water repellent and although the droplet rebounds it has insufficient momentum to separate from the surface, hence remains stuck to the surface. However, if the droplet falls on a superhydrophobic surface with micro-ridges $\left(f_{\mathrm{s}}=0.556, G_{\mathrm{s}}=G_{\mathrm{s} 2}=-0.05\right)$, it bounces off the surface as a distorted sphere but finally comes to rest after several bounces (see Figure 4).

Figure 4 presents the results from simulations of the shape evolution of a droplet impacting an artificial surface constructed from columns of a single microstructure. Droplet shape changes significantly during impact as its kinetic energy is transformed into stored energy resulting from deformation of the droplet surface. Both the springiness in this deformation and the surface hydrophobicity enables the droplet to bounce a few times.

To validate our simulation results, we filmed drops of water bouncing on superhydrophobic surfaces made of micro-pillars. To make these surfaces, we used photolithography and reactive ion etching [24] to create a regular array of square microstructures $(a=4 \mu \mathrm{m})$ on the surface of a silicon wafer; this array of pillars enhances the roughness and produces a superhydrophobic surface. Four different samples were prepared of area fractions $f_{\mathrm{s}}=0.04,0.0625$, 0.1111 and 0.16 , where $f_{\mathrm{s}}$ is defined as $f_{\mathrm{s}}=a^{2} /(a+s)^{2}$ and $s$ is the spacing between the pillars. In sample preparation we used $350 \mu \mathrm{m}$ thick $\langle 100\rangle$ silicon wafer. A fixed pillar height 

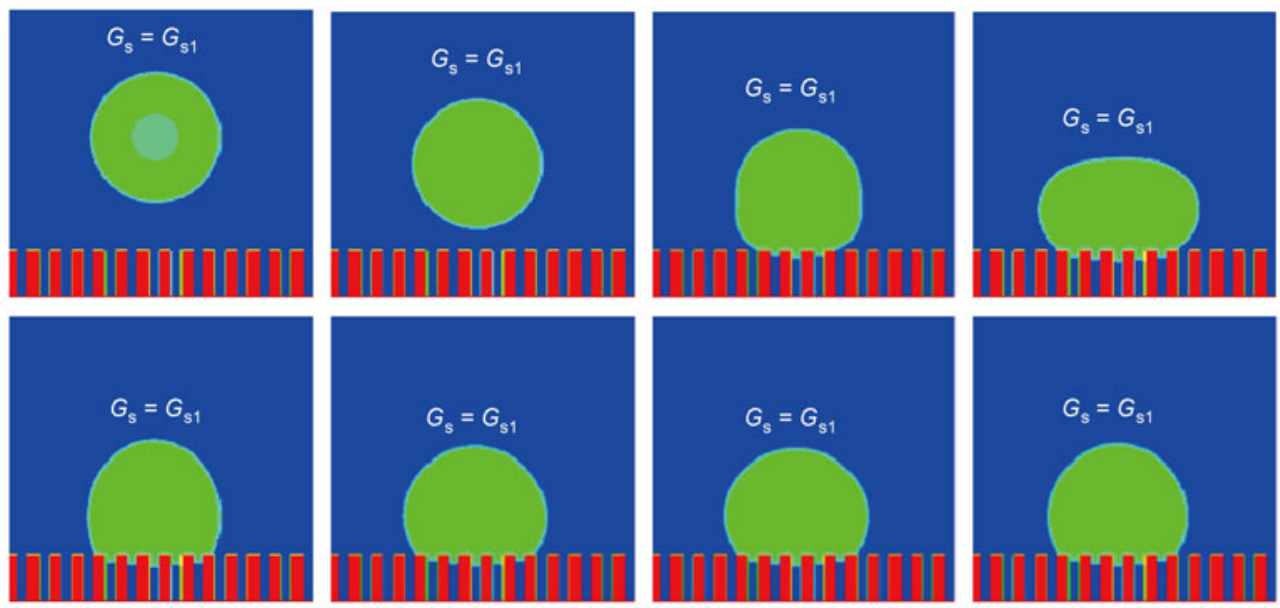

Figure 3 Simulation of water droplet impacting a surface with micro-ridges $\left(f_{\mathrm{s}}=0.556, G_{\mathrm{s}}=G_{\mathrm{s} 1}=-2.9\right)$.
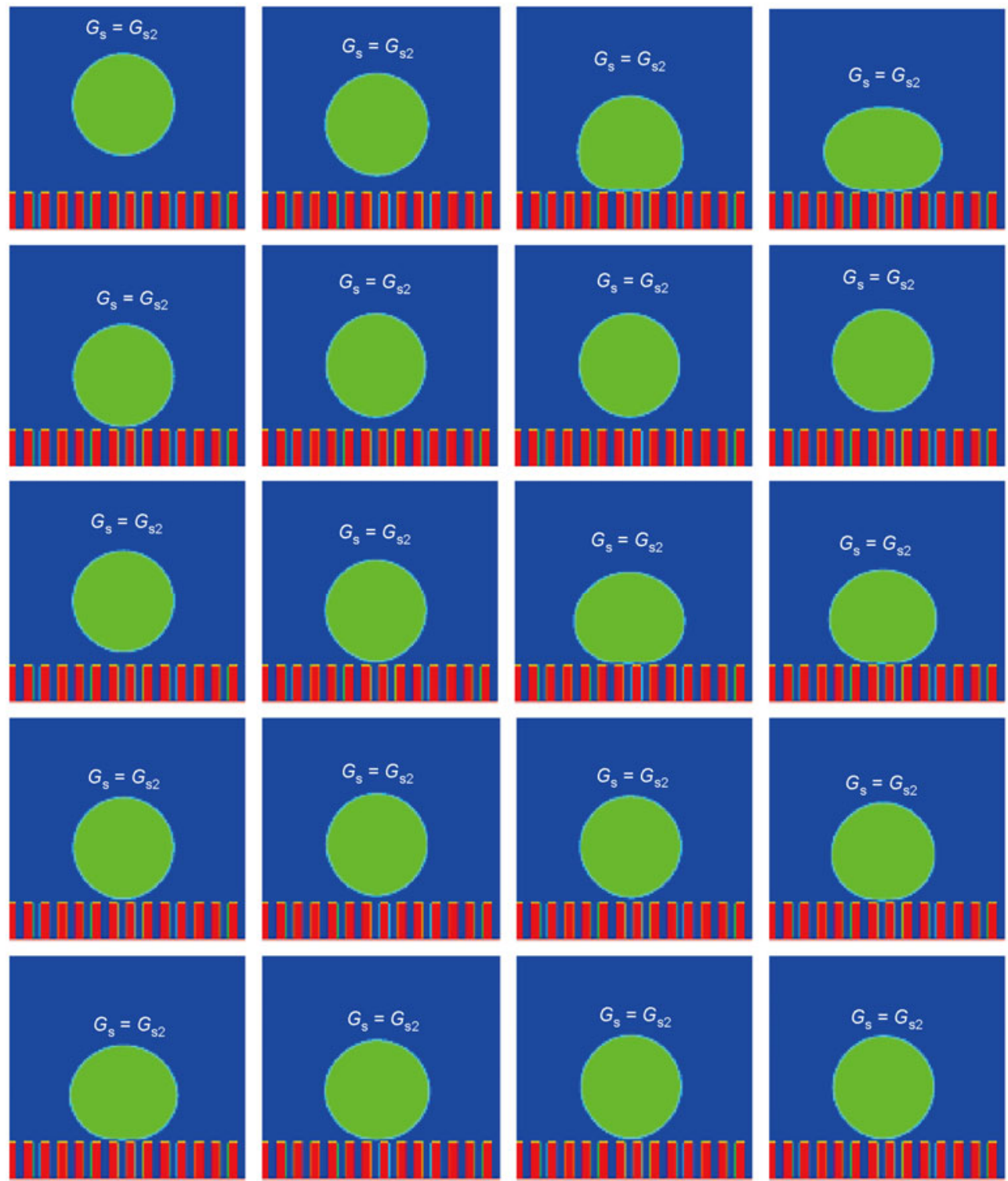

Figure 4 Simulation of a water droplet impacting a surface with micro-ridges $\left(f_{\mathrm{s}}=0.556, G_{\mathrm{s}}=G_{\mathrm{s} 2}=-0.05\right)$. 
$(d=35 \mu \mathrm{m})$ for all the pillar-structured surfaces studied was achieved by controlling the etching time. The surface of the silicon wafer is made hydrophobic by a monolayer covering of a hydrophobic substance; for this purpose we used octadecyltrichlorosilane (OTS) of formula $\mathrm{C}_{18} \mathrm{H}_{37} \mathrm{Cl}_{3} \mathrm{Si}$ (Acros Organics). The surface was first treated with a solution of "Piranha", composed of $70 \%$ sulfuric acid $\left(\mathrm{H}_{2} \mathrm{SO}_{4}\right)$ solution with $98 \%$ concentration and $30 \%$ hydrogen peroxide $\left(\mathrm{H}_{2} \mathrm{O}_{2}\right)$ solution with $20 \%$ concentration. The procedure consists in immersing the surface in the solution of "Piranha" for 30 min at a constant temperature of $90^{\circ} \mathrm{C}$ inside a heater. The surface was then rinsed with deionized water and dried. In a completely dry container, $0.1 \mathrm{~mL}$ of OTS is mixed with 25 $\mathrm{mL}$ of hexadecane. The surface was rinsed with this solution and baked in an oven for $20 \mathrm{~min}$. Then the surface was treated with chloroform for $15 \mathrm{~min}$ to remove any dust, and finally the surface was soaked in anhydrous alcohol for 30 min and dried. After chemical modification, Young's contact angle was measured to be $148^{\circ}$ on the surface with 4 $\mu \mathrm{m}$ pillar and $4 \mu \mathrm{m}$ pillar spacing.

With the various micro-structured hydrophobic surfaces, a photo sequence of a droplet impact was taken with a CCD camera (see Figure 5). Comparing Figures 4 and 5, we see that droplet bouncing in our simulation data is in good agreement with that observed in our experiment. Simulations were also made to investigate flow details inside the droplet. Figure 6 presents two stages of a bouncing droplet on a micro-ridged surface $\left(f_{\mathrm{s}}=0.556,\left|G_{\mathrm{s} 2}\right|<\left|G_{\mathrm{s}}=-1.4\right|<\left|G_{\mathrm{s} 1}\right|\right)$. During each bounce, the droplet deforms and twists from side to side before finally adhering to the surface. The interior flow characteristics under distortion are depicted by velocity vector field. This field is not uniform and varies with time, causing irregular distortions from both top to bottom and side to side.

\subsection{Rebounded height of the droplet}

We investigate how different surface free energy (i.e. different $G_{\mathrm{s}}$ ) affects the maximum height of rebound for droplets of the same size falling through the same height $\left(H_{\text {drop }}\right)$ onto a flat surface. The fractional rebounded height $\left(H_{\mathrm{B}} / H_{\text {drop }}\right)$ is presented in Figure 7 . In simulations with $G_{\mathrm{s}}$ near 0 , the surface free energy is also near zero. If the surface free energy is lower than a critical value, a falling droplet will rebound from a superhydrophobic surface; the lower the free energy, the higher the maximum rebounded height is. Initially, the maximum rebound height of the droplet increases rapidly as surface free energy decreases; further decreases induce an asymptotic decline in maximum rebound height.

We performed similar simulations with a surface of fixed surface free energy $\left(G_{\mathrm{s}}=-0.9\right)$ but varying surface structures. The results for the fractional maximum rebound height $\left(H_{\mathrm{B}} / H_{\text {drop }}\right)$ are presented in Figure 8 . The smooth surface allows no rebounding of the droplet. The surface with dual-scale micro/nano structures with larger static ACA allows a higher rebounded height than for the micro-structure surface. We also investigated responses from different micro-structured surfaces under the same conditions; the fractional maximum rebound heights $\left(H_{\mathrm{B}} / H_{\text {drop }}\right)$ are shown in Figure 9. On surfaces with micro-structures of smaller solid fraction and larger static ACA, the droplet will
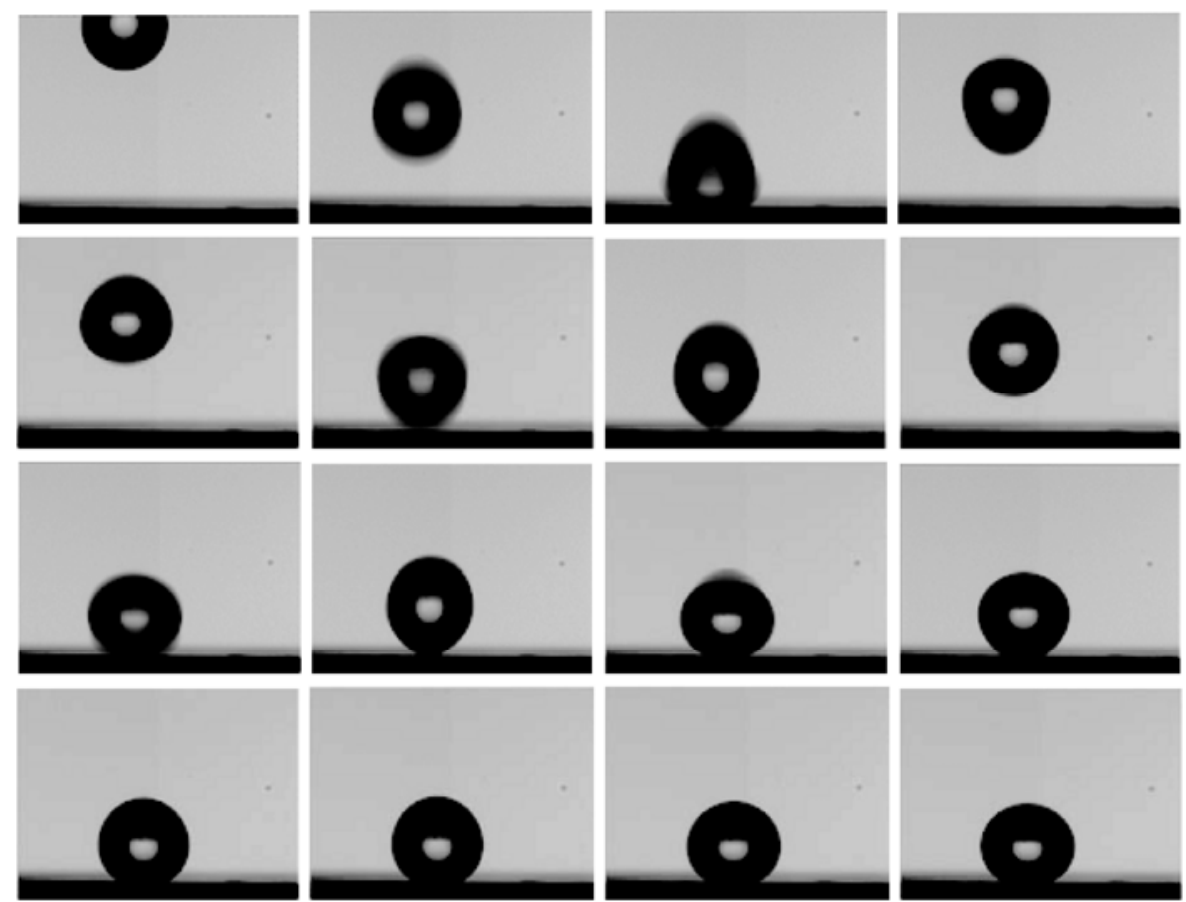

Figure 5 Snapshots of a water droplet impacting on a superhydrophobic surface. 

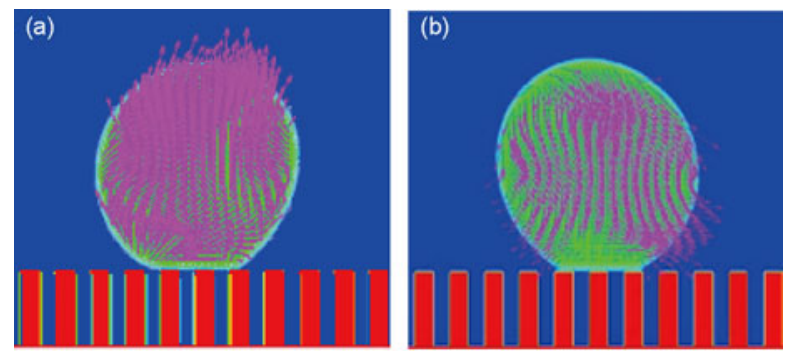

Figure 6 The inside flow characteristics of the droplet at twisting state. (a) Upward distortion; (b) downward distortion.

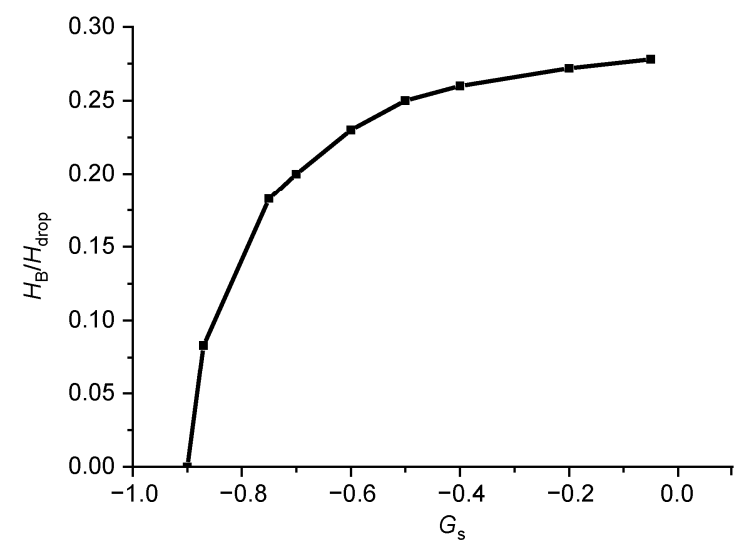

Figure 7 Droplet rebound height from a flat surface as a function of free energy.

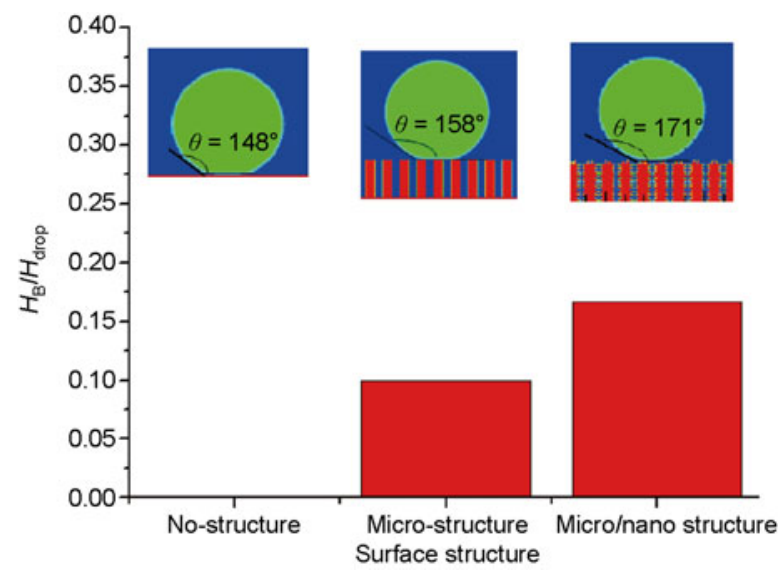

Figure 8 Droplet rebound height from surfaces with different structures $\left(G_{\mathrm{s}}=-0.9\right)$.

have a higher rebounded height; thus, rebound height depends on surface structures. These simulations indicated that hierarchical micro/nano-scale structures do enhance the hydrophobicity of the surface, thus validating those experiments on artificial and natural superhydrophobic materials $[25,26]$.

\section{Conclusion}

In summary, based on the LBM, we investigated the static

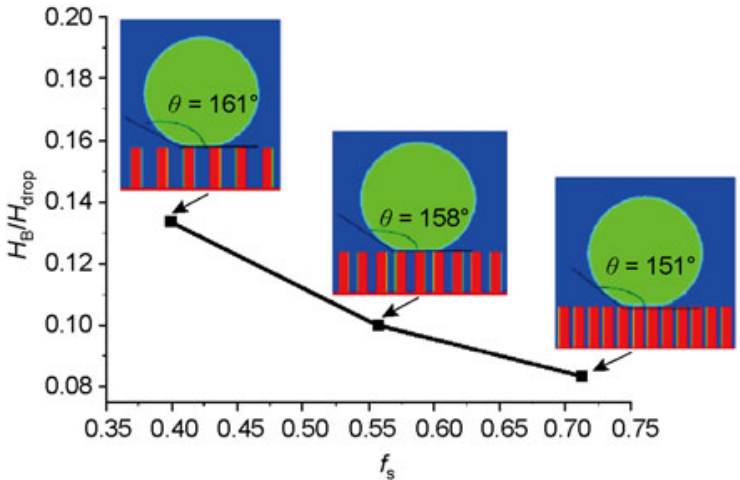

Figure 9 Droplet rebound height from surfaces with different solid fraction micro-structures $\left(G_{\mathrm{s}}=-0.9\right)$.

and dynamic wetting property of water droplets on various hydrophobic surfaces. For ones with micro-structures, we found that the smaller the solid area fraction (i.e. wider micro-ridge spacing), the larger the ACA and the greater the surface hydrophobicity. However, the Cassie state of a droplet on a surface with the smaller solid area fraction would gradually change to a Wenzel state. The surface with dual micro/nano-scale structures not only has an increased surface hydrophobicity, but also stabilized the Cassie state of droplets. Droplets impacting on a superhydrophobic surface rebounded and bounced several times, before finally adhering to the surface.

To the best of our knowledge, this study is the first to investigate, by LBM-based numerical simulations, the static and dynamic hydrophobicity of the superhydrophobic surface with hierarchical roughness. The simulation results are in agreement with our experimental observation. For droplets of the same size falling from the same height, the fractional maximum rebound height depended on the static ACA of the different superhydrophobic surfaces; the lower the free energy, the higher is the rebound height. Moreover, for the same free energy surface, dual micro/nano-scale surface structures increase the rebound height. Consequently, these surface structures could be used in the design of anti/de-ice systems.

This work was supported by the National Natural Science Foundation of China (10872106 and 11072126).

1 Barthlott W, Neinhuis C. Characterization and distribution of waterrepellent, self-cleaning plant surfaces. Ann Bot, 1997, 79: 667-677

2 Cassie A B D, Baxter S. Wettability of porous surface. Trans Faraday Soc, 1944, 40: 546-551

3 Johnson R E, Dettre R H, Brandreth D A. Dynamic contact angles and contact-angles hystresis. J Colloid Interf Sci, 1977, 62: 205-212

4 Kim D, Hwang W, Park H C, et al. Superhydrophobic nano-wire entanglement structures. J Micromech Microeng, 2006, 16: 2593-2597

5 Kim D, Kim J, Park H C, et al. A superhydrophobic dual-scale engineered lotus leaf. J Micromech Microeng, 2008, 18: 015019

6 Feng L, Li H S, Li Y S, et al. Super-hydrophobic surfaces: From natural to artificial. Adv Mater, 2002, 14: 1857-1860

7 Xiu Y, Zhu L, Hess D W, et al. Hierarchical silicon etched structures 
for controlled hydrophobicity/superhydrophobicity. Nano Lett, 2007, 7: 3388-3393

8 Jiang L, Zhao Y, Zhai J. A lotus-leaf-like superhydrophobic surface: A porous microsphere/nanofiber composite film prepared by electrohydrodynamics. Angew Chem Int Ed, 2004, 43: 4338-4341

9 Zhang L, Zhou Z, Cheng B, et al. Superhydrophobic behavior of a perfluoropolyether lotus-leaf-like topography. Langmuir, 2006, 22: $8576-8580$

10 Steele A, Bayer I, Loth E. Inherently superoleophobic nanocomposite coatings by spray atomization. Nano Lett, 2009, 9: 501-505

11 Xu L, Zhang W W, Nagel S R. Drop splashing on a dry smooth surface. Phys Rev Lett, 2005, 94: 184505

$12 \mathrm{Xu} \mathrm{L}$, Barcos L, Nagel S R. Splashing of liquids: Interplay of surface roughness with surrounding gas. Phys Rev E, 2007, 76: 066311

$13 \mathrm{Xu} \mathrm{L}$. Liquid drop splashing on smooth, rough and textured surfaces. Phys Rev E, 2007, 75: 056316

14 Reyssat M, Pépin A, Marty F, et al. Bouncing transitions on microtextured materials. Europhys Lett, 2006, 74: 306-312

15 Chen L, Xiao Z, Chan P C H, et al. Static and dynamic characterization of robust superhydrophobic surfaces built from nano-flowers on silicon micro-post arrays. Micromech Microeng, 2010, 20: 105001

16 Bartolo D, Bouamrirene F, Verneuil E, et al. Bouncing or sticky droplets: Impalement transitions on superhydrophobic micropatterned surfaces. Europhys Lett, 2006, 74: 299-305
17 Renardy M, Renardy Y, Li J. Numerical simulation of moving contact line problems using a volume-of-fluid method. J Comput Phys, 2001, 171: 243-263

18 Spelt P D M A. A level-set approach for simulations of flows with multiple moving contact lines with hysteresis. J Comput Phys, 2005, 207: $389-404$

19 Huang J J, Shu C, Chew Y T. Lattice Boltzmann study of droplet motion inside a grooved channel. Phys Fluids, 2009, 21: 022103

20 Hyvaluoma J, Timonen J. Impalement transitions in droplets impacting microstructured superhydrophobic surfaces. Europhys Lett, 2008, 83: 64002

21 Sukop M C, Thorne D T. Lattice Boltzmann Modeling. Berlin: Springer-Verlag, 2006

22 Succi S. The Lattice Boltmann Equation for Fluid Dynamics and Beyond. Oxford: Clarendon Press, 2001

23 Qian Y H, Succi S, Orszag S A. Recent advances in lattice Boltzmann computing. Ann Rev Comp Phys, 1995, 30: 195-242

24 Hao P F, Yao Z H, Zhang X W. Study of dynamic hydrophobicity of micro-structured hydrophobic surfaces and lotus leaves. Sci China Phys Mech Astron, 2011, 54: 675-682

25 Bhushan B, Nosonovsky M. Hierarchical roughness makes superhydrophobic states stable. Microelectr Eng, 2007, 84: 382-386

$26 \mathrm{Li} \mathrm{W}$, Amirfazli A. Hierarchical structures for natural superhydrophobic surfaces. Soft Matter, 2008, 4: 462-466

Open Access This article is distributed under the terms of the Creative Commons Attribution License which permits any use, distribution, and reproduction in any medium, provided the original author(s) and source are credited. 